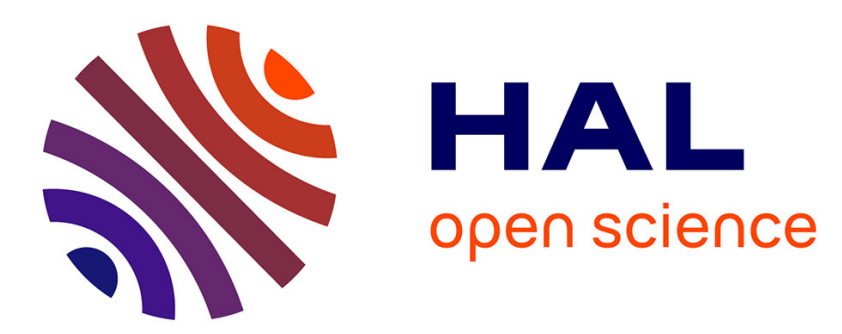

\title{
Existence of Pulses for the System of Competition of Species
}

\author{
Martine Marion, Vitaly Volpert
}

\section{To cite this version:}

Martine Marion, Vitaly Volpert. Existence of Pulses for the System of Competition of Species. Journal of Dynamics and Differential Equations, 2019, 31, pp.1653-1670. 10.1007/s10884-017-9582-6 . hal01573773

\section{HAL Id: hal-01573773 \\ https://hal.science/hal-01573773}

Submitted on 10 Aug 2017

HAL is a multi-disciplinary open access archive for the deposit and dissemination of scientific research documents, whether they are published or not. The documents may come from teaching and research institutions in France or abroad, or from public or private research centers.
L'archive ouverte pluridisciplinaire HAL, est destinée au dépôt et à la diffusion de documents scientifiques de niveau recherche, publiés ou non, émanant des établissements d'enseignement et de recherche français ou étrangers, des laboratoires publics ou privés. 


\title{
Existence of pulses for the system of competition of species
}

\author{
Martine Marion • Vitaly Volpert
}

Received: date / Accepted: date

\begin{abstract}
The paper is concerned with the existence of pulses for monotone reaction-diffusion systems of two equations. For a general class of systems we prove that pulses exist if and only if the wave solutions propagate at positive speed. This result is applied to investigate the existence of pulses for the system of competition of species.
\end{abstract}

Keywords Existence of pulses · Monotone systems · Leray-Schauder method Mathematics Subject Classification (2000) MSC 35K57 · MSC 47F05

\section{Introduction}

We consider the system of two equations

$$
w_{1}^{\prime \prime}+F_{1}\left(w_{1}, w_{2}\right)=0, \quad w_{2}^{\prime \prime}+F_{2}\left(w_{1}, w_{2}\right)=0,
$$

where the function $F=\left(F_{1}, F_{2}\right)$ is assumed to be sufficiently smooth and to satisfy the monotonicity condition:

$$
\frac{\partial F_{i}}{\partial w_{j}}(w)>0 \text { for } i \neq j \text { and } w=\left(w_{1}, w_{2}\right) \in \mathbb{R}_{+}^{2},
$$

together with $F_{1}(0,0)=F_{2}(0,0)=0$.

We aim to study the existence of pulses, that is of positive solutions of system (1) with the limits at infinity $w( \pm \infty)=(0,0)$. The existence of pulses has been proved for some reaction-diffusion systems: the Gray-Scott model [1],

M. Marion

Institut Camille Jordan, UMR 5585 CNRS, Ecole Centrale de Lyon, 69134 Ecully, France

E-mail: martine.marion@ec-lyon.fr

V. Volpert

Institut Camille Jordan, UMR 5585 CNRS, University Lyon 1, 69622 Villeurbanne, France RUDN University, ul. Miklukho-Maklaya 6, Moscow, 117198, Russia 
[4], [13], the Gierer-Meinhardt model [6], [14] and a three component system [2]. Singular perturbation methods to study existence and stability of pulses for a system of two equations are used in [3]. In a previous work [5] we studied the particular case where the nonlinear terms in (1) take the form:

$$
F_{1}\left(w_{1}, w_{2}\right)=f_{1}\left(w_{2}\right)-w_{1}, \quad F_{2}\left(w_{1}, w_{2}\right)=f_{2}\left(w_{1}\right)-w_{2} .
$$

Note that in that case the graph $w_{1}=f_{1}\left(w_{2}\right)$ (respectively $w_{2}=f_{2}\left(w_{1}\right)$ ) corresponds to the zeros of $F_{1}$ (respectively $F_{2}$ ).

Here we aim to consider more general monotone systems. Let us state our assumptions on $F$. For simplicity, we consider infinitely differentiable functions. Setting $w^{+}=(0,0)$ and $w^{-}=(1,1)$ we suppose that

$$
F\left(w^{ \pm}\right)=(0,0),
$$

the eigenvalues of the matrices $F^{\prime}\left(w^{ \pm}\right)$are negative.

Under these conditions $F$ necessarily possesses at least one additional zero in the unit open square and we assume the uniqueness of such a zero, more precisely

$F$ has three zeros $w^{+}, w^{-}, \bar{w}=\left(\bar{w}_{1}, \bar{w}_{2}\right)$ in $\mathbb{R}_{+}^{2}$; furthermore $0<\bar{w}_{i}<1$ and the matrix $F^{\prime}(\bar{w})$ has a positive eigenvalue.

Next we assume that the zeros of $F_{1}$ lie in the graph of some function parametrized by $w_{2}$, more precisely:

$$
F_{1}\left(w_{1}, w_{2}\right)=0, \quad w_{1}, w_{2} \geq 0, \quad \Leftrightarrow \quad w_{1}=f_{1}\left(w_{2}\right),
$$

where

$$
f_{1}(0)=0, \quad f_{1}(1)=1, \quad f_{1}^{\prime}\left(w_{2}\right)>0 \text { for } w_{2} \geq 0 .
$$

Similarly, we assume that the zeros of $F_{2}$ are determined by some function depending on $w_{1}$ :

$$
F_{2}\left(w_{1}, w_{2}\right)=0, \quad w_{1}, w_{2} \geq 0, \quad \Leftrightarrow \quad w_{2}=f_{2}\left(w_{1}\right),
$$

with

$$
f_{2}(0)=0, \quad f_{2}(1)=1, \quad f_{2}^{\prime}\left(w_{1}\right)>0 \text { for } w_{1} \geq 0 .
$$

Under the above conditions we aim to investigate the existence of pulses such that:

$$
w(x)=w(-x), \quad w^{\prime}(x)<0 \text { for } x>0
$$

(here and everywhere below inequalities for vectors mean that each component of the vectors satisfies this inequality). Then the problem on the whole axis reduces to looking for solutions of system (1) defined on the half-axis $\mathbb{R}_{+}$such that

$$
w^{\prime}(0)=0, \quad w^{\prime}(x)<0 \text { for } x>0, \quad w(\infty)=0 .
$$


As already mentioned this problem was studied in [5] for specific nonlinear terms. We showed that the existence of pulses is related to the sign of the wave speed for the traveling-wave solution of the reaction-diffusion system

$$
\frac{\partial v}{\partial t}=\frac{\partial^{2} v}{\partial x^{2}}+F(v)
$$

This solution reads $v(x, t)=u(x-c t)$ where $u$ satisfies the system of equations

$$
u^{\prime \prime}+c u^{\prime}+F(u)=0
$$

and has the limits at infinity

$$
u( \pm \infty)=w^{ \pm}
$$

Under the assumptions (2)-(4), it is classical that there exists a uniquely defined traveling-wave (up to some translation in space for $u$ ). Moreover, it is a monotonically decreasing vector-function (component-wise) (see [7], [8]).

The main result of this work is given by the following theorem.

Theorem 1.1. Under the assumptions (2)-(8), Problem (1) has a solution satisfying (9) if and only if the wave speed $c$ in (11)-(12) is positive.

This result will be applied to investigate the existence of pulses for the system of competition of species. In order to apply the general existence result we will need to introduce some suitable perturbed system. We also discuss conditions which guarantee the positiveness of the speed.

The paper is organized as follows. In Section 2 we first recall some results concerning the functional setting for Problem (1) and some estimates of the solutions borrowed from [5]. Then the existence part in Theorem 1.1 is derived in Section 3. The proof relies on the Leray-Schauder method. We will reduce the nonlinearity to a model problem such that $F_{1}=F_{2}$ and will look for a solution with $w_{1}=w_{2}$. In order to obtain a priori estimates of solutions, we will have to verify that the wave speed remains positive in the process of homotopy. This condition will require some additional constructions. It is worth noting that the homotopy arguments are completely different from the ones in [5]. Finally Section 4 is devoted to the system of competition of pulses.

\section{Functional spaces and estimates of solutions}

Let us consider the system depending on some parameter $\tau \in[0,1]$

$$
\left\{\begin{array}{l}
w_{1}^{\prime \prime}+F_{1}^{\tau}\left(w_{1}, w_{2}\right)=0 \\
w_{2}^{\prime \prime}+F_{2}^{\tau}\left(w_{1}, w_{2}\right)=0
\end{array}\right.
$$


on the half axis $x>0$ with the boundary conditions and the conditions at infinity:

$$
w_{i}^{\prime}(0)=0, \quad w_{i}(\infty)=0, \quad i=1,2 .
$$

For simplicity we will suppose that the function $F^{\tau}=\left(F_{1}^{\tau}, F_{2}^{\tau}\right)$ is infinitely differentiable with respect to both variables $w \in \mathbb{R}^{2}$ and $\tau \in[0,1]$. It satisfies the following conditions that are extensions of (2)-(8):

$$
\frac{\partial F_{i}^{\tau}}{\partial w_{j}}(w)>0, \quad i, j=1,2, \quad i \neq j, \quad w \in \mathbb{R}_{+}^{2}, \quad \tau \in[0,1],
$$

$F^{\tau}\left(w^{ \pm}\right)=(0,0), \quad$ the eigenvalues of the matrices $\left(F^{\tau}\right)^{\prime}\left(w^{ \pm}\right)$are negative,

$F^{\tau}$ has three zeros $w^{+}, w^{-}, \bar{w}^{\tau}=\left(\bar{w}_{1}^{\tau}, \bar{w}_{2}^{\tau}\right)$ in $\mathbb{R}_{+}^{2}$; furthermore $0<\bar{w}_{i}^{\tau}<1$ and the matrix $\left(F^{\tau}\right)^{\prime}\left(\bar{w}^{\tau}\right)$ has a positive eigenvalue,

$$
\begin{aligned}
& F_{1}^{\tau}\left(w_{1}, w_{2}\right)=0 \Leftrightarrow w_{1}=f_{1}^{\tau}\left(w_{2}\right), \quad f_{1}^{\tau^{\prime}}\left(w_{2}\right)>0 \text { for } w_{2} \geq 0, \\
& F_{2}^{\tau}\left(w_{1}, w_{2}\right)=0 \Leftrightarrow w_{2}=f_{2}^{\tau}\left(w_{1}\right), \quad f_{2}^{\tau^{\prime}}\left(w_{1}\right)>0 \text { for } w_{1} \geq 0 .
\end{aligned}
$$

\subsection{Operators and spaces}

For the functional setting let us introduce the Hölder space $C^{k+\alpha}\left(\mathbb{R}_{+}\right)$consisting of vector-functions of class $C^{k}$, which are continuous and bounded on the half-axis $\mathbb{R}_{+}$together with their derivatives of order $k$, and such that the derivatives of order $k$ satisfy the Hölder condition with the exponent $\alpha \in(0,1)$. The norm in this space is the usual Hölder norm. Set

$$
E^{1}=\left\{w \in C^{2+\alpha}\left(\mathbb{R}_{+}\right), w_{i}^{\prime}(0)=0, i=1,2\right\}, \quad E^{2}=C^{\alpha}\left(\mathbb{R}_{+}\right) .
$$

Next we introduce the weighted spaces $E_{\mu}^{1}$ and $E_{\mu}^{2}$ with $\mu(x)=\sqrt{1+x^{2}}$. These spaces are equipped with the norms:

$$
\|w\|_{E_{\mu}^{i}}=\|w \mu\|_{E^{i}}, \quad i=1,2 .
$$

In view of (13), let us consider the operator

$$
A^{\tau}(w)=w^{\prime \prime}+F^{\tau}(w),
$$

acting from $E_{\mu}^{1}$ into $E_{\mu}^{2}$. Then the linearized operator about any function in $E_{\mu}^{1}$ satisfies the Fredholm property and has the zero index. The nonlinear operator is proper on closed bounded sets. This means that the inverse image of a compact set is compact in any closed bounded set in $E_{\mu}^{1}$. Finally, the topological degree can be defined for this operator. All these properties can be found in [10], [11].

Hereafter we consider solutions of (13)-(14) belonging to the space $E_{\mu}^{1}$. 


\subsection{A priori estimates}

We aim to derive various estimates of the solutions of (13)-(14). Note that this problem possesses the trivial solution $w \equiv w^{+}$.

We will be particularly interested in monotonically decreasing solutions. We start with some preliminary properties of such solutions

Lemma 2.1. Under assumptions (15)-(19), assume that $w$ is a monotonically decreasing non trivial solution of (13)-(14). Then for $x>0$ we have $w^{\prime}(x)<0$ and $w(x)<w^{-}$.

This result is a variant of similar ones in [5] (see Lemma 2.3 and the arguments in the proof of Theorem 2.1). The details are left to the reader.

Next we aim to derive estimates in $E_{\mu}^{1}$ of the monotonically decreasing solutions of (13)-(14). They will follow from the positiveness of the wave speed for the traveling wave problem

$$
u^{\prime \prime}+c u^{\prime}+F^{\tau}(u)=0 .
$$

In view of the assumptions (15)-(17), there exists a unique value of $c$ denoted by $c^{\tau}$ such that the system (21) has a monotonically decreasing solution with the limits at infinity:

$$
u( \pm \infty)=w^{ \pm}
$$

The following result is proved in [5].

Theorem 2.2. Under assumptions (15)-(19), moreover suppose that $c^{\tau}>0$ for all $\tau \in[0,1]$. Then there exists some constant $R>0$ such that for all $\tau \in[0,1]$ and for any arbitrary monotonically decreasing solution $w \in E_{\mu}^{1}$ of (13)-(14) the following estimate holds :

$$
\|w\|_{E_{\mu}^{1}} \leq R
$$

Next we state a result of separation between the monotonically decreasing solutions of (13) that will be denoted by $w^{M}(x)$ and the non-monotone solutions of (13) that will be denoted by $w^{N}(x)$.

Theorem 2.3. Suppose that the assumptions (15)-(19) hold true. Then there exists a constant $r>0$ such that for any monotonically decreasing solution $w^{M}$ and any non-monotone solution $w^{N}$ (and for all $\tau \in[0,1]$ ) the following estimates hold:

$$
\left\|w^{M}-w^{N}\right\|_{E_{\mu}^{1}} \geq r, \quad w_{1}^{M}(0)>r, \quad w_{2}^{M}(0)>r .
$$

We refer to [5] for the proof of this result. 


\section{Proof of Theorem 1.1}

The non-existence part in Theorem 1.1 for $c \leq 0$ can be proved as in [5] to which the reader is referred.

Hereafter we assume $c>0$ and we aim to derive the existence of a monotonically decreasing pulse. The proof relies on the Leray-Schauder method. The key step consists in the construction of a continuous deformation (homotopy) of our problem to a model problem for which we will be able to prove that the value of the topological degree is different from zero.

The continuous deformation will take the form (13) and we will require that the conditions (15)-(19) are satisfied. Besides as $\tau$ varies we will need the speed $c^{\tau}$ in (21)-(22) to remain positive. Thanks to Theorem 2.2 this will yield a priori estimates in $E_{\mu}^{1}$ of the monotonically decreasing solutions.

In Section 3.1 we first describe two tools that will allow us to keep the speed $c^{\tau}$ positive and will be used repeatedly. Next the homotopy argument is described in Section 3.2 and we conclude the proof of the existence of a pulse in Section 3.3.

\subsection{Positiveness of the wave speed}

Consider the problem

$$
u^{\prime \prime}+c u^{\prime}+G(u)=0, \quad u( \pm \infty)=w^{ \pm}
$$

Here $G$ is some arbitrary function satisfying the conditions (2)-(8) (with $F=G$ and $f_{i}=g_{i}$ for the zero lines). Then there is a monotone solution of problem (23) for a unique value of the speed $c$. Even though we keep the same notation for the speed it is of course different than the one in (11)-(12). In particular the speed for (23) is not assumed to be positive.

We aim to modify the function $G$ so that the conditions (2)-(8) remain satisfied, the zeros of the nonlinearities remain unchanged and the speed of the traveling wave for the new nonlinearity becomes positive.

For that purpose let us introduce infinitely differentiable and non-negative functions $\phi_{i}(w), i=1,2$, whose supports are defined as follows (Figure 1).

We choose a point $w^{0}=\left(w_{1}^{0}, w_{2}^{0}\right)$ such that $G_{i}\left(w^{0}\right)>0$ for $i=1,2$. Due to the assumptions (2)-(8) such a point necessarily belongs to the zone between the two lines of zeros in the upper part of the square. Also note that $G_{1}\left(w_{1}^{0}, w_{2}\right)>0$ for $w_{2} \geq w_{2}^{0}$ and $G_{2}\left(w_{1}, w_{2}^{0}\right)>0$ for $w_{1} \geq w_{1}^{0}$.

Let us set

$$
D_{1}=\left\{w=\left(w_{1}, w_{2}\right), \quad w_{1}^{0}-\epsilon_{1} \leq w_{1} \leq w_{1}^{0}+\epsilon_{1}, \quad w_{2}^{0}-\epsilon_{2} \leq w_{2} \leq 1\right\},
$$



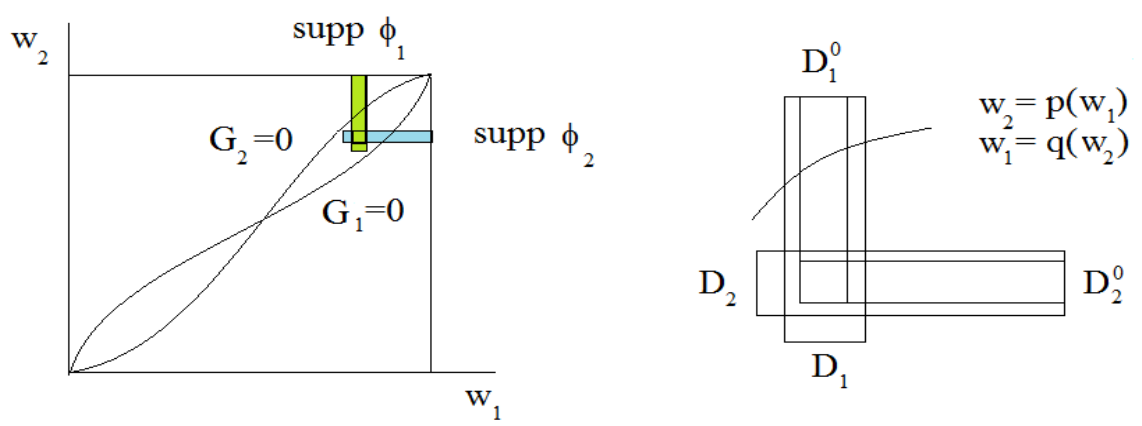

Fig. 1 Zero lines of the functions $G_{i}$ and the supports of the functions $\phi_{i}$ (left). More detailed structure of the support of the functions $\phi_{i}$ (right). Inner rectangles show where these functions equal 1 .

where $\epsilon_{1}$ and $\epsilon_{2}$ are positive numbers and sufficiently small so that $G_{1}(w)>0$ for $w \in D_{1}$. Similarly, we introduce

$$
D_{2}=\left\{w=\left(w_{1}, w_{2}\right), \quad w_{2}^{0}-\delta_{1} \leq w_{2} \leq w_{2}^{0}+\delta_{1}, \quad w_{1}^{0}-\delta_{2} \leq w_{1} \leq 1\right\},
$$

where $\delta_{1}$ and $\delta_{2}$ are sufficiently small positive numbers so that $G_{2}(w)>0$ for $w \in D_{2}$.

Next, we can introduce two rectangles $D_{i}^{0}, i=1,2$, with common width $\sigma$ located inside the rectangles $D_{i}$ as it is shown in Figure 1 (right). We define the functions $\phi_{i}$ in such a way that the following conditions are satisfied:

$$
\frac{\partial \phi_{1}}{\partial w_{2}} \geq 0, \quad \frac{\partial \phi_{2}}{\partial w_{1}} \geq 0
$$

and

$$
\phi_{i} \geq 0, \quad \operatorname{Supp} \phi_{i} \subset D_{i}, \quad \phi_{i}(w)=1 \text { for } w \in D_{i}^{0}, \quad i=1,2 .
$$

We now consider the nonlinearities:

$$
H_{i}(w)=G_{i}(w)+k \phi_{i}(w), \quad i=1,2,
$$

where $k$ is some positive constant. The function $H_{i}, i=1,2$, differs from $G_{i}$ in a zone where $G_{i}>0$ (see Figure 1 ) and it is obtained by adding some positive term. Consequently the zeros of $H_{i}$ coincide with the ones of $G_{i}$ and $H_{i}(w)>0$ at any point $w$ such that $G_{i}(w)>0$. The conditions (3)-(8) remain valid for $H$ and the monotonicity condition

$$
\frac{\partial H_{i}}{\partial w_{j}}(w)>0, \quad i \neq j,
$$

is satisfied. 
Hence the traveling wave problem for this modified nonlinearity which reads:

$$
u^{\prime \prime}+c u^{\prime}+G(u)+k \phi(u)=0, \quad u( \pm \infty)=w^{ \pm},
$$

where we set $\phi(u)=\left(\phi_{1}(u), \phi_{2}(u)\right)$ has a monotone solution for a unique value of the speed. This value depends on $k$ and we denote it by $c_{k}$. We aim to show that if $k$ is large enough then $c_{k}>0$.

Lemma 3.1. Let $G$ be a function satisfying the conditions (2)-(8) and let $w^{0}$ such that $G\left(w^{0}\right)>0$. Define the rectangles $D, D_{i}^{0}$ (with width $\sigma$ ) and the function $\phi_{i}$ as above. Then if

$$
k>\frac{\|G\|_{\infty}}{\sigma}
$$

the speed $c_{k}$ in (28) is positive.

Proof. Let $u(x)=\left(u_{1}(x), u_{2}(x)\right)$ denote the solution of (28). Since both components of the solution are monotone functions of $x$, we can express one coordinate as a function of the other one: either $u_{2}=p\left(u_{1}\right)$ or $u_{1}=q\left(u_{2}\right)$. Both of them are monotone functions of their arguments.

Next consider the solution $u(x)$ as a curve in the plane $\left(w_{1}, w_{2}\right)$. It connects the points $w^{+}$and $w^{-}$. Therefore it crosses the set $D^{0}=D_{1}^{0} \cup D_{2}^{0}$ (Figure 2, right). Moreover necessarily the curve crosses the whole width of either $D_{1}^{0}$ or $D_{2}^{0}$.

Without loss of generality we can suppose that the curve crosses the whole width of $D_{1}^{0}$. Then let us consider the function $u_{2}=p\left(u_{1}\right)$ along the curve. The system (28) reduces to the single equation

$$
u_{1}^{\prime \prime}+c_{k} u_{1}^{\prime}+G_{1}\left(u_{1}, p\left(u_{1}\right)\right)+k \phi_{1}\left(u_{1}, p\left(u_{1}\right)\right)=0 .
$$

For this scalar equation it is easy to see that

$$
c_{k}=\frac{1}{\left\|u_{1}^{\prime}\right\|_{L^{2}(-\infty, \infty)}^{2}} \int_{0}^{1}\left[G_{1}(s, p(s))+k \phi_{1}(s, p(s))\right] d s .
$$

Hence $c_{k}>0$ if and only if

$$
\int_{0}^{1}\left[G_{1}(s, p(s))+k \phi_{1}(s, p(s))\right] d s>0 .
$$

Since the function $\phi_{1}$ is non-negative and $\phi_{1}=1$ on $D_{1}^{0}$, we have

$$
\int_{0}^{1} \phi_{1}(s, p(s)) d s>\sigma
$$

where $\sigma$ is the width of the rectangle $D_{1}^{0}$. Consequently the inequality (31) is satisfied if the stronger condition

$$
\int_{0}^{1} G_{1}(s, p(s)) d s+k \sigma>0
$$


holds true. This last condition is clearly satisfied under the assumption (29) (where $\|G\|_{\infty}$ denotes the supremum norm of $G$ on the unit square). This implies that $c_{k}>0$ and concludes the proof of the lemma.

We will also make use of a classical result that states that the speed of the waves increases if the nonlinearity is increased (see [8]).

Theorem 3.2. Consider the two problems

$$
u^{\prime \prime}+c u^{\prime}+G^{k}(u)=0, \quad u( \pm \infty)=w^{ \pm}, \quad k=1,2,
$$

where the functions $G^{k}=\left(G_{1}^{k}, G_{2}^{k}\right)$ are assumed to satisfy conditions (2) (possibly non-strict) and (3), and furthermore

$$
G_{i}^{1}(u) \geq G_{i}^{2}(u) \text { for } w_{+} \leq u \leq w_{-} \text {and } i=1,2 .
$$

If the two problems (33) possess monotone solutions with the values of the speed $c=c_{k}, k=1,2$, then we have $c_{1} \geq c_{2}$.

\subsection{Homotopy}

We aim now to construct the homotopy $F^{\tau}$. It is of course such that, for $\tau=0$, $F^{0}=F$ the given nonlinearity in (1) for which we recall that the wave speed $c$ is assumed to be positive.

As already noted we need the speed $c^{\tau}$ for the traveling wave problem (21)(22) to remain positive in the homotopy process. For that purpose we introduce some (arbitrary) point $w^{0} \in(0,1)^{2}$ such that $F\left(w^{0}\right)>0$ and consider the function $\phi=\left(\phi_{1}, \phi_{2}\right)$ constructed as above in Section 3.1 (with $G=F$ ). We will require the homotopy to be such that $F^{\tau}\left(w^{0}\right)$ remains positive as $\tau$ varies so that Lemma 3.1 will apply with $G=F^{\tau}$ and $\phi$ defined as above.

Step $1\left(\tau \in\left[0, \tau_{1}\right], \tau_{1}=1 / 4\right)$. We start by increasing the function $F$ and introduce:

$$
F_{i}^{\tau}(w)=F_{i}(w)+\tau K \phi_{i}(w), \quad i=1,2, \quad 0 \leq \tau \leq \tau_{1}
$$

Here $K$ is some arbitrary positive constant which will be specified below.

As noted in Section 3.1 the functions $F_{i}^{\tau}$ satisfy the conditions (15)-(19) and there zero lines coincide with the ones of $F_{i}$, that is $w_{1}=f_{1}\left(w_{2}\right)$ and $w_{2}=f_{2}\left(w_{1}\right)$.

Let $c^{\tau}$ denote the value of the speed for the problem

$$
u^{\prime \prime}+c^{\tau} u^{\prime}+F^{\tau}(u)=0, \quad u( \pm \infty)=w_{ \pm} .
$$

Since $F_{i}^{\tau} \geq F_{i}$, Theorem 3.2 yields that $c^{\tau} \geq c>0$ for $\tau \in\left[0, \tau_{1}\right]$ (without any assumption on $K$ ). 
Step $2\left(\tau \in\left[\tau_{1}, \tau_{2}\right], \tau_{2}=1 / 2\right)$. Let us introduce the functions:

$$
\bar{F}_{1}(w)=f_{1}\left(w_{2}\right)-w_{1}, \quad \bar{F}_{2}(w)=f_{2}\left(w_{1}\right)-w_{2} .
$$

The second step of the homotopy will consist in reducing the functions $F_{i}^{\tau_{1}}$ to the functions $\bar{F}_{i}$ while keeping $K$ fixed. Hence we consider

$$
F_{i}^{\tau}(w)=\frac{\tau-\tau_{1}}{\tau_{2}-\tau_{1}} \bar{F}_{i}(w)+\frac{\tau_{2}-\tau}{\tau_{2}-\tau_{1}} F_{i}(w)+\tau_{1} K \phi_{i}(w), \quad i=1,2, \quad \tau_{1} \leq \tau \leq \tau_{2} .
$$

The above functions satisfy the monotonicity condition (15) and their zero lines are still given by $w_{1}=f_{1}\left(w_{2}\right)$ and $w_{2}=f_{2}\left(w_{1}\right)$.

Also $F^{\tau}\left(w^{0}\right)>0$. Hence, due to Lemma 3.1 , the wave speed $c^{\tau}$ in (35) is positive for $\tau \in\left[\tau_{1}, \tau_{2}\right]$ if $K$ is sufficiently large.

Step $3\left(\tau \in\left[\tau_{2}, \tau_{3}\right], \tau_{3}=3 / 4\right)$. At the end of the step 2 we obtained

$$
F_{1}^{\tau_{2}}(w)=f_{1}\left(w_{2}\right)-w_{1}+\tau_{1} K \phi_{1}(w), \quad F_{2}^{\tau_{2}}(w)=f_{2}\left(w_{1}\right)-w_{2}+\tau_{1} K \phi_{2}(w) .
$$

We now aim to reduce the two functions $f_{1}$ and $f_{2}$ in (38) to some common value $f_{0}$ while keeping the other terms unchanged. Hence the homotopy will take the form

$$
\begin{gathered}
F_{1}^{\tau}(w)=f_{1}^{\tau}\left(w_{2}\right)-w_{1}+\tau_{1} K \phi_{1}(w), \\
F_{2}^{\tau}(w)=f_{2}^{\tau}\left(w_{1}\right)-w_{2}+\tau_{1} K \phi_{2}(w), \quad \tau_{2} \leq \tau \leq \tau_{3},
\end{gathered}
$$

where $f_{i}^{\tau_{2}}=f_{i}$ and $f_{1}^{\tau_{3}}=f_{2}^{\tau_{3}}=f_{0}$.

In order to satisfy the conditions (15)-(17) we will require that

$$
f_{i}^{\tau^{\prime}}(s)>0 \text { for } s \geq 0, \quad i=1,2 .
$$

The system $w_{1}=f_{1}^{\tau}\left(w_{2}\right), w_{2}=f_{2}^{\tau}\left(w_{1}\right)$ has three solutions $w^{+}, w^{-}, \bar{w}^{\tau}$ in $\mathbb{R}_{+}^{2}$, furthermore $0<\bar{w}_{i}^{\tau}<1$.

$$
f_{1}^{\tau^{\prime}}(0) f_{2}^{\tau^{\prime}}(0)<1, \quad f_{1}^{\tau^{\prime}}(1) f_{2}^{\tau^{\prime}}(1)<1, \quad f_{1}^{\tau^{\prime}}\left(\bar{w}_{2}^{\tau}\right) f_{2}^{\tau^{\prime}}\left(\bar{w}_{1}^{\tau}\right)>1 .
$$

These conditions yield that the graphs of the two functions $f_{1}^{\tau}$ and $f_{2}^{\tau}$ are located with respect to each other as represented in Figure 1. Besides we ask that

$$
F^{\tau}\left(w^{0}\right)>0
$$

so that the speed of the wave in (35) is positive if $K$ is sufficiently large.

Finally in preparation of the step 4 below we require that the function $f_{0}=f_{1}^{\tau_{3}}=f_{2}^{\tau_{3}}$ moreover satisfies

The equation $f_{0}(s)=s$ has three solutions : $0,1, \mu ; 0<\mu<1$,

$$
\int_{0}^{1}\left(f_{0}(s)-s\right) d s>0
$$


We claim that we can define the functions $f_{i}^{\tau}$ in such a way that all the above conditions are satisfied. Let us describe this homotopy in more details.

Recall that the system of equations $w_{1}=f_{1}\left(w_{2}\right), w_{2}=f_{2}\left(w_{1}\right)$, has a single solution $\left(\bar{w}_{1}, \bar{w}_{2}\right)$ satisfying the inequalities $0<\bar{w}_{i}<1, i=1,2$. Let us introduce the inverse function of $f_{1}$ denoted by $\tilde{f}_{1}$. Then the equation $f_{2}(s)=\tilde{f}_{1}(s)$ has a single solution $\bar{w}_{1}$ in the interval $(0,1)$.

Now consider a function $h_{2}$ such that

$$
\begin{gathered}
h_{2}(0)=0, \quad h_{2}\left(\bar{w}_{1}\right)=f_{2}\left(\bar{w}_{1}\right), \quad h_{2}(1)=1, \\
h_{2}(s)>f_{2}(s) \text { for } \bar{w}_{1}<s<1 ; \quad h_{2}(s)<f_{2}(s) \text { for } 0<s<\bar{w}_{1} .
\end{gathered}
$$

Next for $\theta \in[0,1]$ introduce the function:

$$
h_{2}^{\theta}=(1-\theta) f_{2}+\theta h_{2} .
$$

Then the equation $h_{2}^{\theta}(s)=g_{1}(s)$ has exactly three solutions in $[0,1]$, namely 0,1 and $\bar{w}_{1}$.

An explicit form of the function $h_{2}$ can be given by:

$$
h_{2}(s)=\left\{\begin{array}{l}
\epsilon s \text { for } 0 \leq s<a, \\
\frac{1}{\epsilon}\left(s-\bar{w}_{1}\right) \text { for } a \leq s \leq b, \\
\epsilon(s-1)+1 \text { for } b \leq s \leq 1,
\end{array}\right.
$$

where $\epsilon>0$ and $a, b$ are obtained by requiring the continuity of $h_{2}$ at these points. Then if $\epsilon$ is sufficiently small the functions $f_{1}$ and $h_{2}^{\theta}$ satisfy the conditions (40)-(43) (with $f_{1}^{\tau}=f_{1}$ and $f_{2}^{\tau}=h_{2}^{\theta}$ ) except at the points where $h_{2}^{\theta}$ is not differentiable but we can use smoothing procedure to make it infinitely differentiable and satisfy the conditions everywhere.

The above procedure allowed to reduce the function $f_{2}$ to $h_{2}$ with the conditions (40)-(43) being satisfied. Next similarly, we reduce $f_{1}$ to the piecewise linear function $h_{1}$ with

$$
h_{1}(s)=\left\{\begin{array}{l}
\epsilon s \text { for } 0 \leq s<r \\
\frac{1}{\epsilon}\left(s-\bar{w}_{2}\right) \text { for } r \leq s \leq d, \\
\epsilon(s-1)+1 \text { for } d \leq s \leq 1
\end{array}\right.
$$

(up to smoothing)

At this point, in the homotopy process, the couple $\left(f_{1}, f_{2}\right)$ became $\left(h_{1}, h_{2}\right)$ which are piece-wise linear function depending on $\epsilon>0, \bar{w}_{1}$ and $\bar{w}_{2}$ (up to smoothing). Now in the definition of $h_{1}$ and $h_{2}$ we replace the point $\bar{w}$ by by $\alpha \bar{w}$ with $\alpha \in[0,1]$. Then if $\epsilon$ and $\alpha$ are sufficiently small the conditions (40)-(43) remain valid and moreover

$$
\int_{0}^{1}\left(h_{1}(s)-s\right) d s>0, \quad \int_{0}^{1}\left(h_{2}(s)-s\right) d s>0 .
$$

Note that either $h_{1} \geq h_{2}$ or $h_{2} \geq h_{1}$. For sufficiently small $\epsilon$ and $\alpha$ let us consider the function $f_{0}$ which is the maximum of these two functions. The homotopy of the functions $h_{1}$ to $f_{0}$ (or $h_{2}$ to $f_{0}$ ) can be easily constructed in such a way that the conditions on the homotopy are verified. 
Step $4\left(\tau \in\left[\tau_{3}, 1\right]\right)$. In this last step, we eliminate the functions $\phi_{i}$ by setting:

$$
F_{i}^{\tau}(w)=f_{0}\left(w_{j}\right)-w_{i}+\tau_{1} \frac{1-\tau}{1-\tau_{3}} K \phi_{i}(w), \quad i=1,2 \text { and } j \neq i, \quad \tau_{3} \leq \tau \leq 1 .
$$

Note that $F_{i}^{\tau}$ decreases as $\tau$ increases. Hence by Theorem 3.2 the speed of the corresponding traveling wave decreases. However it remains larger than the one for the limit problem corresponding to $\tau=1$. This problem reads

$$
u^{\prime \prime}+c u^{\prime}+F^{1}(u)=0, \quad u( \pm \infty)=w^{ \pm},
$$

with

$$
F^{1}(u)=\left(f_{0}\left(u_{2}\right)-u_{1}, f_{0}\left(u_{1}\right)-u_{2}\right) .
$$

It possesses a uniquely defined solution such that $u_{1}=u_{2}$. The corresponding speed $c^{1}$ has the sign of the integral in (45) which is positive. Hence for $\tau \in$ $\left[\tau_{3}, 1\right]$ we conclude that $c^{\tau} \geq c^{1}>0$.

\subsection{Existence of solutions}

We have constructed the homotopy from our original problem to the system

$$
w_{1}^{\prime \prime}+f_{0}\left(w_{2}\right)-w_{1}=0, \quad w_{2}^{\prime \prime}+f_{0}\left(w_{1}\right)-w_{2}=0,
$$

on the half axis $x>0$ with the boundary condition and the condition at infinity:

$$
w^{\prime}(0)=0, \quad w(\infty)=0 .
$$

Let us look for some solution of problem (49)-(50) for which $w_{1} \equiv w_{2}$ so that $w_{1}$ satisfies

$$
w_{1}^{\prime \prime}+f_{0}\left(w_{1}\right)-w_{1}=0, w_{1}^{\prime}(0)=0, w_{1}(\infty)=0 .
$$

Due to the properties of $f_{0}$ and in particular (45) it is easily seen that (51) possesses a unique solution satisfying $w_{1}^{\prime}(x)<0$ for $x>0$.

We can now prove the existence of solutions of problem (1) satisfying (9).

In Section 3.2 we constructed the homotopy $F^{\tau}$. Let us now consider the spaces introduced in Section 2 and the operator $A^{\tau}$ given by (20).

Since $c^{\tau}>0$ for $\tau \in[0,1]$, it satisfies the conditions imposed to obtain a priori estimates of monotonically decreasing solutions (Theorem 2.2). Denote by $B \subset E_{\mu}^{1}$ a ball which contains all monotonically decreasing solutions. Since the operator $A^{\tau}$ is proper on closed bounded sets with respect to both variables $w$ and $\tau$, the set of monotonically decreasing solutions of the problem $A^{\tau}(w)=$ 0 is compact. Since they are separated from non-monotone solutions (Theorem 2.3), then we can construct a domain $D \subset B \subset E_{\mu}^{1}$ such that all monotonically decreasing solutions (for all $\tau \in[0,1]$ ) are located inside $D$ and there are no other solutions in the closure $\bar{D}$. Indeed it is sufficient to take a union of small 
balls of the radius $r$ (Theorem 2.3) around each monotonically decreasing solution.

Let us note that only strictly monotone solutions belong to this domain. In particular, the trivial solution $w \equiv 0$ does not belong to it since the monotonically decreasing solutions are separated from the trivial solution (Theorem 2.3). This is an important remark because the index of the trivial solution equals one. If it belongs to the domain $D$, then the sum of the indices, which equals the value of the degree, can be zero (see below). If it was the case, then we could not affirm the existence of solutions for the original problem.

We can now determine the topological degree $\gamma\left(A^{\tau}, D\right)$. We have

$$
\gamma\left(A^{0}, D\right)=\gamma\left(A^{1}, D\right)=\sum_{j} \text { ind } u^{j},
$$

where the sum is taken with respect to all monotonically decreasing solutions $u^{j}$ of problem (49)-(50), and ind $u^{j}$ denotes the index of the solution. We have ind $u^{j}=(-1)^{\nu_{j}}$, where $\nu_{j}$ is the number of positive eigenvalues of the linearized problem. We proved in [5] that $\nu_{j}=1$ so that ind $u^{j}=-1$. Hence the degree is different from zero for the original problem, and the equation $A^{0}(w)=0$ has a solution in $D$. This concludes the proof of the existence result in Theorem 1.1

Let us note that we used the existence of solutions of the model problem but not their uniqueness. The index of each monotone solution equals -1 . Therefore the degree is different from zero for any number of solutions if at least one such solution exists.

\section{The model of competition of species}

We consider the system of equations

$$
W_{1}^{\prime \prime}+W_{1}\left(1-W_{1}-\alpha W_{2}\right)=0, \quad W_{2}^{\prime \prime}+\rho W_{2}\left(1-\beta W_{1}-W_{2}\right)=0,
$$

describing the competition of species in population dynamics. The unknowns are two population densities and the nonlinear expressions represent the rates of reproduction of the two species. All parameters are positive constants and we will suppose that $\alpha>1$ and $\beta>1$.

Then the system has four stationary points: $P_{0}=(0,0), P_{1}=(1,0), P_{2}=$ $(0,1)$ and $P_{3}=\left(\bar{W}_{1}, \bar{W}_{2}\right)$ where $0<\bar{W}_{i}<1$ satisfy $\bar{W}_{1}+\alpha \bar{W}_{2}=1$ and $\beta \bar{W}_{1}+\bar{W}_{2}=1$. The points $P_{1}$ and $P_{2}$ are stable while the points $P_{0}$ and $P_{3}$ are unstable.

The above system is not monotone. However it is possible to obtain a monotone system by introducing the new variable $w_{1}=W_{1}$ and $w_{2}=1-W_{2}$. The system of equations now reads:

$$
w_{1}^{\prime \prime}+F_{1}\left(w_{1}, w_{2}\right)=0, \quad w_{2}^{\prime \prime}+F_{2}\left(w_{1}, w_{2}\right)=0,
$$


where

$$
\begin{gathered}
F_{1}\left(w_{1}, w_{2}\right)=w_{1}\left(1-w_{1}-\alpha\left(1-w_{2}\right)\right), \\
F_{2}\left(w_{1}, w_{2}\right)=\rho\left(1-w_{2}\right)\left(\beta w_{1}-w_{2}\right) .
\end{gathered}
$$

These functions satisfy the conditions

$$
\frac{\partial F_{1}}{\partial w_{2}}\left(w_{1}, w_{2}\right)>0, \quad \frac{\partial F_{2}}{\partial w_{1}}\left(w_{1}, w_{2}\right)>0,
$$

if $w_{1}>0$ and $w_{2}<1$.

We will also need to consider the system of equations

$$
U_{1}^{\prime \prime}+c U_{1}^{\prime}+U_{1}\left(1-U_{1}-\alpha U_{2}\right)=0, \quad U_{2}^{\prime \prime}+c U_{2}^{\prime}+\rho U_{2}\left(1-\beta U_{1}-U_{2}\right)=0,
$$

describing travelling wave solutions of the system of competition of species. We will look for its solutions with the limits at infinity

$$
U_{1}(\infty)=0, \quad U_{2}(\infty)=1, \quad U_{1}(-\infty)=1, \quad U_{2}(-\infty)=0 .
$$

The equivalent problem in terms of the variables $u_{1}=U_{1}$ and $u_{2}=1-U_{2}$ reads

$$
u_{1}^{\prime \prime}+c u_{1}^{\prime}+u_{1}\left(1-\alpha-u_{1}+\alpha u_{2}\right)=0, \quad u_{2}^{\prime \prime}+c u_{2}^{\prime}+\rho\left(1-u_{2}\right)\left(\beta u_{1}-u_{2}\right)=0,
$$

with the limits :

$$
u_{1}(\infty)=u_{2}(\infty)=0, \quad u_{1}(-\infty)=u_{2}(-\infty)=1 .
$$

Existence and uniqueness of travelling wave, that is of solutions of problem (57)-(58) (or equivalently (59)-(60)) is known (note that the inequality (56) is not strict on the closed unit square, but the result still holds, see [8]).

Hereafter we will denote by $c_{0}$ the corresponding wave speed. The wave speed $c_{0}$ can be positive, zero or negative depending on the values of parameters. If it is positive the wave propagates in the direction of the values $\left(U_{1}, U_{2}\right)=(1,0)$ reached at $-\infty$. Some elements on the sign of the wave speed can be found in Section 4.2.

4.1 Existence of pulses for Problem (53)-(56)

\subsubsection{Pulses for a perturbed system}

As already mentioned the nonlinearity in (53) is such that the system is monotone. However the conditions on the zeros lines stated in (5)-(8) are not satisfied (see Figure 2 below). This leads us to introduce the following modified functions involving the parameter $\epsilon>0$ :

$$
\left\{\begin{array}{c}
F_{1}^{\epsilon}\left(w_{1}, w_{2}\right)=w_{1}\left(1-\alpha-w_{1}+\alpha w_{2}\right)+\epsilon \alpha w_{2}, \\
F_{2}^{\epsilon}\left(w_{1}, w_{2}\right)=\rho\left(1-w_{2}\right)\left(\beta w_{1}-w_{2}\right)-\epsilon \rho w_{2} .
\end{array}\right.
$$


For these new nonlinearities the equations $F_{1}^{\epsilon}\left(w_{1}, w_{2}\right)=0$ and $F_{2}^{\epsilon}\left(w_{1}, w_{2}\right)=$ 0 are respectively equivalent to

$$
w_{2}=f_{1}^{\epsilon}\left(w_{1}\right) \text { and } w_{1}=f_{2}^{\epsilon}\left(w_{2}\right),
$$

where

$$
f_{1}^{\epsilon}\left(w_{1}\right)=\frac{-w_{1}\left(1-\alpha-w_{1}\right)}{\alpha\left(\epsilon+w_{1}\right)}, \quad f_{2}^{\epsilon}\left(w_{2}\right)=\frac{\left(1-w_{2}+\epsilon\right) w_{2}}{\beta\left(1-w_{2}\right)} .
$$

For all $\epsilon>0$ sufficiently small the functions $f_{1}^{\epsilon}$ and $f_{2}^{\epsilon}$ are monotone functions of their respective arguments (Figure 2), and the system of equations

$$
w_{2}=f_{1}^{\epsilon}\left(w_{1}\right), \quad w_{1}=f_{2}^{\epsilon}\left(w_{2}\right),
$$

possesses three solutions. Let us denote the two exterior points by $w_{ \pm}^{\epsilon}$ and the intermediate point by $\bar{w}^{\epsilon}$. We have $w_{+}^{\epsilon}=(0,0)$ and $w_{-}^{\epsilon} \rightarrow(1,1)$ as $\epsilon \rightarrow 0$.
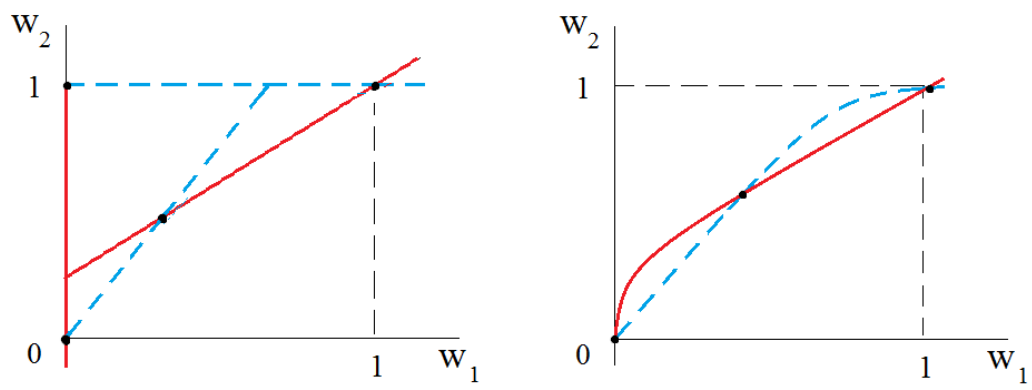

Fig. 2 Left: zeros of the functions $F_{1}$ (bold solid) and $F_{2}$ (bold dashed). Right: zeros of the functions $F_{1}^{\epsilon}$ (bold solid) and $F_{2}^{\epsilon}$ (bold dashed).

For the perturbed nonlinearities the pulse is the solution of the system:

$$
w_{1}^{\prime \prime}+F_{1}^{\epsilon}\left(w_{1}, w_{2}\right)=0, \quad w_{2}^{\prime \prime}+F_{2}^{\epsilon}\left(w_{1}, w_{2}\right)=0, \quad w_{i}( \pm \infty)=0, \quad i=1,2,
$$

while the traveling wave problem reads:

$$
u_{1}^{\prime \prime}+c u_{1}^{\prime}+F_{1}^{\epsilon}\left(u_{1}, u_{2}\right)=0, \quad u_{2}^{\prime \prime}+c u_{2}^{\prime}+F_{2}^{\epsilon}\left(u_{1}, u_{2}\right)=0, \quad u( \pm \infty)=w_{ \pm}^{\epsilon} .
$$

The existence of travelling waves for the perturbed system is investigated in $[12]$ where the following result is derived.

Proposition 4.1. For all $\epsilon>0$ sufficiently small Problem (63) possesses a solution for a unique value of $c$ denoted by $c_{\epsilon}$. Moreover, $c_{\epsilon}$ is a continuous function of $\epsilon$.

Let us note that the limiting value $c_{0}$ in Proposition 4.1 is the speed of the travelling wave for the system (59)-(60). In particular if $c_{0}>0$, then $c_{\epsilon}>0$ for 
all positive and sufficiently small $\epsilon$. This remark enables us to apply Theorem 1.1 to derive the existence of pulses for the perturbed problem for sufficiently small $\epsilon$.

Proposition 4.2. Assume that $c_{0}>0$. Then, for all $\epsilon>0$ sufficiently small, Problem (62) possesses a solution $w_{\epsilon}$ such that

$$
w_{\epsilon}^{\prime}(0)=0, \quad w_{\epsilon}^{\prime}(x)<0 \text { for } x>0, \quad w_{\epsilon}(\infty)=0 .
$$

\subsubsection{Pulses for the original problem}

The existence of some pulse for Problem (53) is related to the sign of the speed $c_{0}$ as stated in the following result.

Theorem 4.3. System (53) has a solution satisfying

$$
w^{\prime}(0)=0, \quad w^{\prime}(x)<0 \text { for } x>0, \quad w(\infty)=0
$$

if and only if the speed $c_{0}$ in problem (59)-(60) is positive.

Proof. Again we refer to [5] for the proof of the non-existence part in Theorem 4.3 for $c_{0} \leq 0$.

Hereafter we assume $c_{0}>0$ and we aim to derive the existence of a pulse. This will proved by taking the limit $\epsilon \rightarrow 0$ for the solutions $w_{\epsilon}$ provided by Proposition 4.2 .

Since $0<w_{\epsilon}(x)<w_{-}^{\epsilon}$ and $w_{-}^{\epsilon} \rightarrow(1,1)$ as $\epsilon \rightarrow 0$, the functions $w_{\epsilon}$ are bounded in $L^{\infty}(\mathbb{R})$ independently of $\epsilon$ sufficiently small. Hence classical regularity estimates yield that the Hölder norm in $C^{2+\alpha}\left(\mathbb{R}_{+}\right)$of $w_{\epsilon}$ is also bounded independently of $\epsilon$ sufficiently small.

Consequently there exists a subsequence still denoted by $w_{\epsilon}$ locally convergent to some limiting function $\widehat{w}$. The function $\widehat{w}$ satisfies the equations (53), $\widehat{w}(x) \geq 0$ and $\widehat{w}^{\prime}(x) \leq 0$ for $x \geq 0, \widehat{w}^{\prime}(0)=0$.

Let us first check that

$$
\widehat{w}(0) \neq(0,0) .
$$

Arguing by contradiction suppose that $w_{\epsilon}(0)$ converges to the origin as $\epsilon \rightarrow 0$. Recalling the definition (61) it is easy to check the existence of some neighborhood of the origin in $\mathbb{R}_{+}^{2}$ such that for all $\epsilon$ sufficiently small and for each point $w>0$ in this neighborhood at least one component of $F^{\epsilon}(w)$ is negative. Due to its convergence property, $w_{\epsilon}(0)$ enters this neighborhood and at least one component of $F^{\epsilon}\left(w_{\epsilon}(0)\right)$ is negative. Then due to the equations the second derivative at $x=0$ of the corresponding component of $w_{\epsilon}$ is positive, and this function cannot be decreasing. Similar arguments provide

$$
\widehat{w}(0) \neq(0,1), \quad \widehat{w}(0) \neq(1,0) .
$$

Next let us verify that

$$
\widehat{w}(0)>(0,0) .
$$


If this is not true then either $\widehat{w}_{1}(0)=0$ and $\widehat{w}_{2}(0)>0$ or $\widehat{w}_{1}(0)>0$ and $\widehat{w}_{2}(0)=0$. In the first case $\widehat{w}_{1}(x) \equiv 0$ and $\widehat{w}_{2}$ satisfies

$$
\widehat{w}_{2}^{\prime \prime}+F_{2}\left(0, w_{2}\right)=0, \quad \widehat{w}_{2} \geq 0, \quad \widehat{w}_{2}^{\prime} \leq 0, \quad \widehat{w}_{2}^{\prime}(0)=0 .
$$

Since $F_{2}\left(0, w_{2}\right) \leq 0$ this provides readily $\widehat{w}_{2}^{\prime}=0$ and $\widehat{w}_{2} \equiv c_{2}$. The constant $c_{2}$ should be such that $c_{2}>0$ and $\left(0, c_{2}\right)$ is a zero of $F$. The only possibility is $c_{2}=1$ but this is impossible since we noted that $\widehat{w}(0) \neq(0,1)$. In the second case similar arguments provide $\left(\widehat{w}_{1}, \widehat{w}_{2}\right) \equiv(1-\alpha, 0)$ which is also impossible since this point is not a zero of $F$ This shows (65).

The above arguments yield the existence of some constant $\eta>0$ such that for $\epsilon$ sufficiently small the two components of $w_{\epsilon}$ satisfy:

$$
w_{\epsilon, 1}(0)>\eta, \quad w_{\epsilon, 2}(0)>\eta
$$

(indeed otherwise there exists a sequence $w_{\epsilon}$ converging to some $\widehat{w}$ and at least one component of $\widehat{w}(0)$ vanishes $)$.

In order to verify the convergence of $\widehat{w}$ to the origin as $x \rightarrow \infty$, it is sufficient to prove that the functions $w_{\epsilon}$ are bounded independently of $\epsilon>$ 0 sufficiently small in the (weighted) norm of $E_{\mu}^{1}$. Since they are uniformly bounded in the Hölder norm without weight, it is sufficient to prove that the norm $\sup _{x}\left|w_{\epsilon}(x) \mu(x)\right|$ is uniformly bounded. Let us recall that solutions $w_{\epsilon}$ converge to zero exponentially at infinity. So this norm is bounded for each solution. Suppose that these functions are not uniformly bounded with respect to $\epsilon$ in the weighted norm. Then there is a sequence of solutions $w_{\epsilon_{k}}$ (with $\left.0<w_{\epsilon_{k}}(x)<w_{-}^{\epsilon_{k}}\right)$ for which

$$
\sup _{x \geq 0}\left|w_{\epsilon_{k}}(x) \mu(x)\right| \rightarrow \infty \text { as } k \rightarrow \infty .
$$

Let $\delta>0$ be sufficiently small so that the exponential decay of the solutions gives the estimate

$$
\left|w_{\epsilon_{k}}(x) \mu(x)\right| \leq M
$$

for those values of $x$ such that $\left|w_{\epsilon_{k}}(x)\right| \leq \delta$, with some constant $M>0$ independent of $k$. Choosing $\delta<\eta$ where $\eta$ is given by (66), we can select $x_{k}$ so that $\left|w_{\epsilon_{k}}\left(x_{k}\right)\right|=\delta$ and

$$
\left|w_{\epsilon_{k}}(x) \mu(x)\right| \leq M \text { for } x \geq x_{k} .
$$

If the values $x_{k}$ are uniformly bounded, then the values $\left|w_{\epsilon_{k}}(x) \mu(x)\right|$ are uniformly bounded for $0 \leq x \leq x_{k}$ since $w_{\epsilon_{k}}(x) \leq w_{\epsilon_{k}}$. Together with (67), this provides the required estimate for all $x \geq 0$.

Suppose that $x_{k} \rightarrow \infty$. Consider the sequence of functions $u^{k}(x)=w_{\epsilon_{k}}(x+$ $\left.x_{k}\right)$. We can choose a subsequence converging to some limiting function $\widehat{u}$ in $C_{l o c}^{2}(\mathbb{R})$. Then $\widehat{u}$ is a non increasing function defined on the whole axis and satisfies the equation

$$
\widehat{u}^{\prime \prime}+F(\widehat{u})=0
$$


The bound (67) yields that $\widehat{u}(\infty)=(0,0)$ while $\widehat{u}(-\infty)=u^{-}$, where $u^{-}$is such that $F\left(u^{-}\right)=0$ and $u^{-} \neq(0,0)$ since $|\widehat{u}(0)|=\delta$. Hence we obtain a solution of system (59) with $c=0$. Let us show that this is not possible.

Indeed, if $u^{-}$is the unstable zero of $F$ in $\left(w^{+}, w^{-}\right)$, then a solution of system (21) exists only for negative $c$ [7], [8]. Let us note, that there are two unstable zeros, $w_{1}=0, w_{2}=1$ and $w_{1}=(1-\alpha) /(1-\alpha \beta), w_{2}=\beta w_{1}$. If $u^{-}=w^{-}$(stable point), then by virtue of the assumption of Theorem 4.3, $c>0$. Hence the function $\widehat{u}$ can not exist, and the sequence $x_{k}$ is bounded.

There remains to show that $\widehat{w}^{\prime}(x)<0$ for all $x>0$ (component-wise). Indeed, suppose that $\widehat{w}_{i}^{\prime}\left(x_{0}\right)=0$ for some $i$ and for some $x_{0}>0$. Assuming for example $i=1$ we differentiate the first equation of system (53). Setting $\widehat{v}=-\widehat{w}_{1}^{\prime}$ it provides :

$$
-\widehat{v}^{\prime \prime}-a(x) \widehat{v}=b(x),
$$

where

$$
a(x)=\frac{\partial F_{1}}{\partial w_{1}}(\widehat{w}), \quad b(x)=-\frac{\partial F_{1}}{\partial w_{2}}(\widehat{w}) \widehat{w}_{2}^{\prime}(x) .
$$

Since $b(x) \geq 0, \widehat{v}(x) \geq 0$ and $\widehat{v}\left(x_{0}\right)=0$, the positiveness theorem yields $\widehat{v}(x) \equiv 0$ hence $\widehat{w}_{1}(x) \equiv 0$. As already noted this implies $\widehat{w}_{2}^{\prime} \equiv 0$ hence $\widehat{w}_{2} \equiv 0$ which is impossible.

The proof of Theorem 4.3 is completed.

Remark. Systems (53) and (52) are equivalent thanks to some appropriate change of variable. Hence Theorem 4.3 provides the existence of some even solution $W$ of system (52) satisfying

$$
W_{1}( \pm \infty)=0, \quad W_{2}( \pm \infty)=1, \quad W_{1}(x)>0 \text { and } W_{2}(x)<1 \text { for } x \in \mathbb{R}
$$

if and only if $c_{0}>0$. Similarly, if $c_{0}<0$, we obtain the existence of some even solution of $(52)$ such that:

$$
W_{1}( \pm \infty)=1, \quad W_{2}( \pm \infty)=0, \quad W_{1}(x)<1 \text { and } W_{2}(x)>0 \text { for } x \in \mathbb{R} \text {. }
$$

Indeed let us compare these two cases. In the first one, after the change of variables $w_{1}=W_{1}, w_{2}=1-W_{2}$ we considered the solution of (53) with zero limits at infinity. It is proved that it exists if and only if the wave in (59) with the limits $u_{i}(-\infty)=1, u_{i}(\infty)=0$ has a positive speed. In terms of the original variables, these limits are given by (see (58)):

$$
U_{1}(-\infty)=1, U_{1}(\infty)=0, \quad U_{2}(-\infty)=0, U_{2}(\infty)=1 .
$$

If we consider a solution of (52) with limits (69), then the change of variables is different and reads: $\tilde{w}_{1}=1-W_{1}, \tilde{w}_{2}=W_{2}$. A study similar to the one for the first change of variable can be carried out. In particuler, the $\tilde{w}_{i}$ have required to have zero limits at infinity. From the analogous theorem, we conclude that such solution exists if and only if the corresponding wave with the limits 
$\tilde{u}_{i}(-\infty)=1, \tilde{u}_{i}(\infty)=0, i=1,2$ has a positive speed. In terms of the original variables, these limits read:

$$
U_{1}(-\infty)=0, U_{1}(\infty)=1, \quad U_{2}(-\infty)=1, U_{2}(\infty)=0
$$

The wave with such limits has a positive speed if and only if the speed of the wave with the limits $(70)$ is negative. Indeed, it is sufficient to replace $U_{i}(x)$ by $U_{i}(-x), i=1,2$. Thus, depending on the sign of the speed, either there is a solution with limits (68) or a solution with limits (69) (and no solutions if $\left.c_{0}=0\right)$.

4.2 The sign of the wave speed

We proved the existence of pulses when the wave speed $c_{0}$ in problem (57)(58) (or equivalently (59)-(60)) is positive. Here we aim to present some simple conditions on the parameters $\rho, \alpha$ and $\beta$ which guarantee that $c_{0}>0$.

We start with a case where $c_{0}=0$ that will be useful afterwards.

Lemma 4.4. Suppose $\rho=1$ and $\alpha=\beta$. Then the speed $c_{0}$ of the wave solution of (57)-(58) is equal to zero.

Proof. Let us argue by contradiction and suppose $c_{0}>0$ (the case $c_{0}<0$ is similar). The functions $z_{1}(x)=U_{2}(-x)$ and $z_{2}(x)=U_{1}(-x)$ satisfy the problem

$$
\begin{gathered}
z_{1}^{\prime \prime}+c^{*} z_{1}^{\prime}+z_{1}\left(1-z_{1}-\alpha z_{2}\right)=0, \quad z_{2}^{\prime \prime}+c^{*} z_{2}^{\prime}+\rho z_{2}\left(1-\alpha z_{1}-z_{2}\right)=0, \\
z_{1}(\infty)=0, \quad z_{2}(\infty)=1, \quad z_{1}(-\infty)=1, \quad z_{2}(-\infty)=0
\end{gathered}
$$

where $c^{*}=-c_{0}<0$. Since the problem (72)-(73) coincides with the problem (57)-(58), then we conclude that the latter has two different waves, one with a positive speed and another one with a negative speed. We obtain a contradiction with the uniqueness of the wave

Our main result is the following one.

Theorem 4.5. If $\rho=1$ and $\beta>\alpha$, then the speed $c_{0}$ of the wave solution of (57)-(58) is positive.

Proof. For $\rho, \alpha$ and $\beta$ satisfying the above conditions let us set

$$
F_{1}\left(u_{1}, u_{2}\right)=u_{1}\left(1-u_{1}-\alpha\left(1-u_{2}\right)\right), \quad F_{2}\left(u_{1}, u_{2}\right)=\left(1-u_{2}\right)\left(\beta u_{1}-u_{2}\right),
$$

and

$$
G_{1}\left(u_{1}, u_{2}\right)=u_{1}\left(1-u_{1}-\alpha\left(1-u_{2}\right)\right), \quad G_{2}\left(u_{1}, u_{2}\right)=\left(1-u_{2}\right)\left(\alpha u_{1}-u_{2}\right) .
$$


Applying Lemma 4.4 to the function $G$ we see that the speed of the wave solution of

$$
u^{\prime \prime}+c u^{\prime}+G(u)=0, \quad u( \pm \infty)=w_{ \pm},
$$

equals zero. Hereafter we denote by $\tilde{u}$ some solution of

$$
\tilde{u}^{\prime \prime}+G(\tilde{u})=0, \quad \tilde{u}( \pm \infty)=w_{ \pm}
$$

(the solution is unique up to space translation). Next since $\beta>\alpha$ we have

$$
F_{1}(u)=G_{1}(u) \text { and } F_{2}(u)>G_{2}(u) \text { for } 0<u_{i}<1 .
$$

Hence we can apply Theorem 3.2 to the functions $F$ and $G$ and we conclude that $c_{0} \geq 0$.

We now aim to show that $c_{0}>0$. Arguing by contradiction let us suppose that $c_{0}=0$. Consider the Cauchy problem

$$
\frac{\partial v}{\partial t}=\frac{\partial^{2} v}{\partial x^{2}}+F(v)
$$

with the initial condition $v(x, 0)=\tilde{u}(x)$, where $\tilde{u}$ is a solution of problem (74). Since

$$
\tilde{u}^{\prime \prime}+F(\tilde{u})=F(\tilde{u})-G(\tilde{u}) \geq 0
$$

(the inequality is strict for the second component of the vector), $\tilde{u}$ is a lower function and $v(x, t)$ increases as a function of $t$ for each $x$ fixed. By virtue of the global asymptotic stability of travelling waves for monotone systems, $v(x, t)$ converges to a solution $u(x)$ of problem (57)-(58). Hence

$$
u(x)>\tilde{u}(x), \quad \forall x \in \mathbb{R} .
$$

For any $h \in \mathbb{R}$ the function $\tilde{u}(x-h)$ is also a solution of problem (74). If $h$ is sufficiently large, then the inequality

$$
u(x)>\tilde{u}(x-h)
$$

does not hold for some $x$. Let $h_{0}$ be the supremum of the $h$ for which (78) holds for all $x \in \mathbb{R}$. If $u(x) \geq \tilde{u}\left(x-h_{0}\right)$ for all $x$ and $u\left(x_{0}\right)=\tilde{u}\left(x_{0}-h_{0}\right)$ for some $x_{0}$, then we obtain a contradiction with the positiveness theorem. We will now consider the case where such finite $x_{0}$ does not exist and shows that this case also leads to some contradiction.

According to the assumptions above, there a sequence $h_{n}$ such that $h_{n}>$ $h_{0}, h_{n} \rightarrow h_{0}$ as $n \rightarrow \infty$ and $u\left(x_{n}\right)=\tilde{u}\left(x_{n}-h_{n}\right)$ for some $x_{n}$ and for one of the components of the solutions. The sequence $x_{n}$ can not be bounded. Consider the case where $x_{n} \rightarrow \infty$.

Let us take some $x^{*}$ such that $F\left(u\left(x^{*}\right)\right)<0$ and some $x_{n}>x^{*}$. Such $x^{*}$ exists since $u(x) \sim p \exp (-\lambda x)$ where $p>0$ is the eigenvector of the matrix $F^{\prime}(0)$ corresponding to the negative eigenvalue, $\lambda$ is a positive number. We consider the boundary value problem for the equation (76) on the halfaxis $x>x^{*}$ with the boundary condition $v\left(x^{*}, t\right)=u\left(x^{*}\right)$. It has a unique stationary solution $u(x)$. Moreover, this solution is globally asymptotically 
stable. Indeed, the initial condition $v(x, 0)=u\left(x^{*}\right)$ provides an upper solution and the initial condition $v(x, 0)=0$ a lower solution. Both of them converge to the stationary solution $u(x)$ by virtue of its uniqueness.

On the other hand, the function $\max \left(u(x), \tilde{u}\left(x-h_{n}\right)\right)$ is a lower function. Therefore the corresponding solution is greater than $u(x)$ and it cannot converge to $u(x)$ since it increases in time. This contradiction proves that $x_{n}$ cannot tend to infinity. Similarly, it can be proved that it cannot go to $-\infty$. This contradiction proves the theorem.

\section{Acknowledgements}

The second author was partially supported by the Ministry of Education and Science of the Russian Federation (the agreement number 02.a03.21.0008) and by the program PICS CNRS 6583 Matbio.

\section{References}

1. A. Doelman, R. A. Gardner, T. J. Kaper, Stability analysis of singular patterns in the 1-D Gray-Scott model, Physica D, 122, 1-36 (1998).

2. A. Doelman, P. van Heijster, T. J. Kaper, Pulse dynamics in a three-component system: existence analysis, J. Dyn. Diff. Equat., 21, 73-115 (2009).

3. A. Doelman, F. Veerman. An explicit theory for pulses in two component, singularly perturbed, reaction-diffusion equations. Journal of Dynamics and Differential Equations, 27 no. 3, 555-595 (2015).

4. J.K. Hale, L.A. Peletier, W.C. Troy, Exact homoclinic and heteroclinic solutions of the Gray-Scott model for autocatalysis, SIAM J. Appl. Math., 61, 102-130 (2000).

5. M. Marion, V. Volpert, Existence of pulses for a monotone reaction-diffusion system, Pure and Applied Funct. Analysis, 1, 97-122 (2016).

6. Y. Nec, M.J. Ward, The stability and slow dynamics of two-spike patterns for a class of reaction-diffusion system, Math. Model. Nat. Phenom., 8, 206-232 (2013).

7. A.I. Volpert, V.A. Volpert, Applications of the rotation theory of vector fields to the study of wave solutions of parabolic equations, Trans. Moscow Math. Soc., 52, 59-108 (1990).

8. A. Volpert, Vit. Volpert, Vl. Volpert, Travelling wave solutions of parabolic systems, Translation of Mathematical Monographs, Vol. 140, Amer. Math. Society, Providence, 1994.

9. V. Volpert, A. Volpert, Spectrum of elliptic operators and stability of travelling waves, Asymptotic Analysis, 23, 111-134 (2000).

10. V. Volpert, A. Volpert, Properness and topological degree for general elliptic operators, Abstract and Applied Analysis, 129-182 (2003).

11. V. Volpert, Elliptic partial differential equations. Volume 1. Fredholm theory of elliptic problems in unbounded domains, Birkhäuser, 2011.

12. V. Volpert, Elliptic partial differential equations. Volume 2. Reaction-diffusion equations, Birkhäuser, 2014.

13. J. Wei, M. Winter, Stability of spiky solutions in a reaction-diffusion system with four morphogens on the real line, SIAM J. Math. Anal., 42, 2818-2841 (2010).

14. J. Wei, M. Winter, Existence, classification and stability analysis of multiple-peaked solutions for the Gierer-Meinhardt system in $\mathbb{R}^{1}$, Methods Appl. Anal.,14, no. 2, 119163 (2007). 